\section{The combination of spatial frequency and orientation is effortlessly perceived}

\section{DOV SAGI \\ The Weizmann Institute of Science, Rehovot, Israel}

It is known that two stimuli can be distinguished in parallel if they differ with respect to certain features, but not if they contain identical features in different combinations and differ merely with respect to feature conjunctions (Bergen \& Julesz, 1983; Nakayama \& Silverman, 1986; Treisman \& Gelade, 1980). This paradigm is an attractive means of determining those features of a stimulus that the visual system detects in parallel. However, the list of "parallel features" obtained in this way conflicts, at least in part, with a similar list of features that are registered early, which derives from other, more venerable psychophysical methods (Houk \& Hoffman, 1987). Two stimulus dimensions in particular-spatial frequency and orientation-exemplify this conflict. Experiments using the Treisman paradigm (search tasks) indicate that spatial frequency and orientation are independent parallel features because conjunctions between these features cannot be discriminated in parallel (Walters, Biederman, \& Weisstein, 1983). In contrast, experiments using threshold and masking techniques indicate the existence of parallel channels that are selective with respect to both spatial frequency and orientation of a stimulus (Watson, 1983; Wilson, 1983).

The present research note aims to resolve this conflict. It is suggested that a failure to effortlessly (i.e., in parallel) perceive a target in the standard Treisman display may result from local differences between the distractors used. Figure 1 demonstrates the problem for target elements that differ with respect to spatial frequency and orientation. The display contains 49 patches, within which luminance varies as a Gabor function. Approximately half the patches are of low frequency and horizontal orientation (Lh), and an approximately equal number are of high frequency and vertical orientation $(\mathrm{Hv})$. The task is to detect one patch, which may occur anywhere, with low frequency and vertical orientation (Lv). According to Treisman, the $\mathrm{Lv}$ patch cannot be detected in parallel, since doing so requires a search in two separate feature dimensions (that of spatial frequency and that of orientation). This reasoning rests on two assumptions, the first being that spatial frequency and orientation are the relevant dimensions, and the second, that detection fails only for conjunctions. However, recent findings indicate that detection fails

This work was supported by grants from the Israel Center for Psychobiology and the B. De Rothschild Foundation for the Advancement of Science in Israel, Inc. Thanks are extended to Jochen Braun for his helpful comments on the manuscript. The author's mailing address is Department of Applied Mathematics and Computer Sciences, The Weizmann Institute of Science, Rehovot 76100, Israel. equally in the absence of prominent feature gradients and in the presence of irrelevant gradients (Nothdurft, 1985; Sagi \& Julesz, 1985, 1987). These studies lead to the conclusion that only differences between patches are registered in parallel, although the properties that give rise to these differences are not detected (Sagi \& Julesz, 1985). The consequence relevant in the present context is that gradients of different types cannot be distinguished in parallel: The $L v$ patch differs in one dimension (either frequency or orientation) from all its neighbors, whereas all other patches ( $\mathrm{Lh}$ or $\mathrm{Hv}$ ) differ from an average of half their neighbors in two dimensions (both frequency and orientation). Thus, the average feature gradient is uniform over the entire display, and parallel detection of the Lv patch may fail simply because it does not present a prominent gradient.

To determine whether this is, in fact, why the $\mathrm{Lv}$ patch is not detected in parallel, a display with no gradients between distractor patches can be used (Figure 2). In Figure 2, luminance within a patch varies as the sum of two Gabor functions. The distractor patches were generated by overlapping the distractor patches ( $\mathrm{Lh}$ and $\mathrm{Hv}$ ) in Figure 1, and the target patch was obtained by summing two patches with the opposite combinations ( $\mathrm{L} v$ and $\mathrm{Hh}$ ). To detect the target, combinations of spatial frequency and orientation must be compared. Both the target and the distractor contain components of both horizontal and vertical orientation, and of both low and high spatial frequency. The difference lies solely in the way orientation and spatial frequency are combined. In distinction to Figure 1, however, the target in Figure 2 is effortlessly perceived (and this is true for all contrast ratios of the two frequencies). It is easy to devise analogous demonstrations for other stimulus dimensions. For the dimensions of color and orientation, one may use a target composed of a red vertical line and a blue horizontal line embedded in distractors composed of blue vertical and red horizontal lines. For these two dimensions, however, detection of the target requires sequential inspection of individual elements.

A demonstration in the spirit of the Treisman paradigm thus confirms that information about spatial frequency and orientation is registered jointly, as has long been claimed in studies of spatial frequency and orientation channels, which thus appear to provide the basis for parallel detection of this combined information. As for color, information about color and orientation appears to be carried by separate channels, thus preventing parallel detection. Note that the manner in which spatial frequency and orientation are combined in a channel does not imply detection of a conjunction. Channels summate linearly over a range of spatial frequencies and orientations, and their response is not restricted to a single conjunction ( $\mathrm{L}$ and $v$, for example). A final point to note is the assumption that feature gradients are pooled locally. This pooling 
A

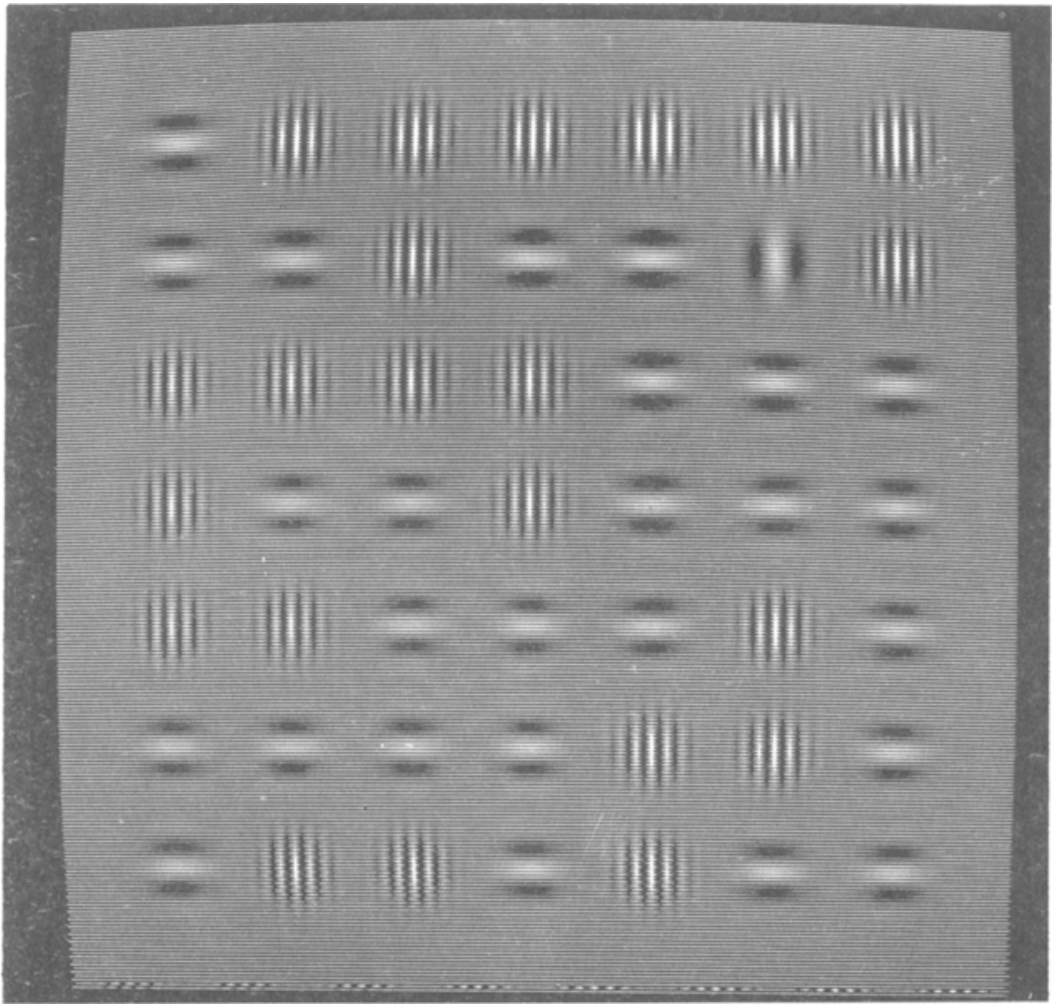

B

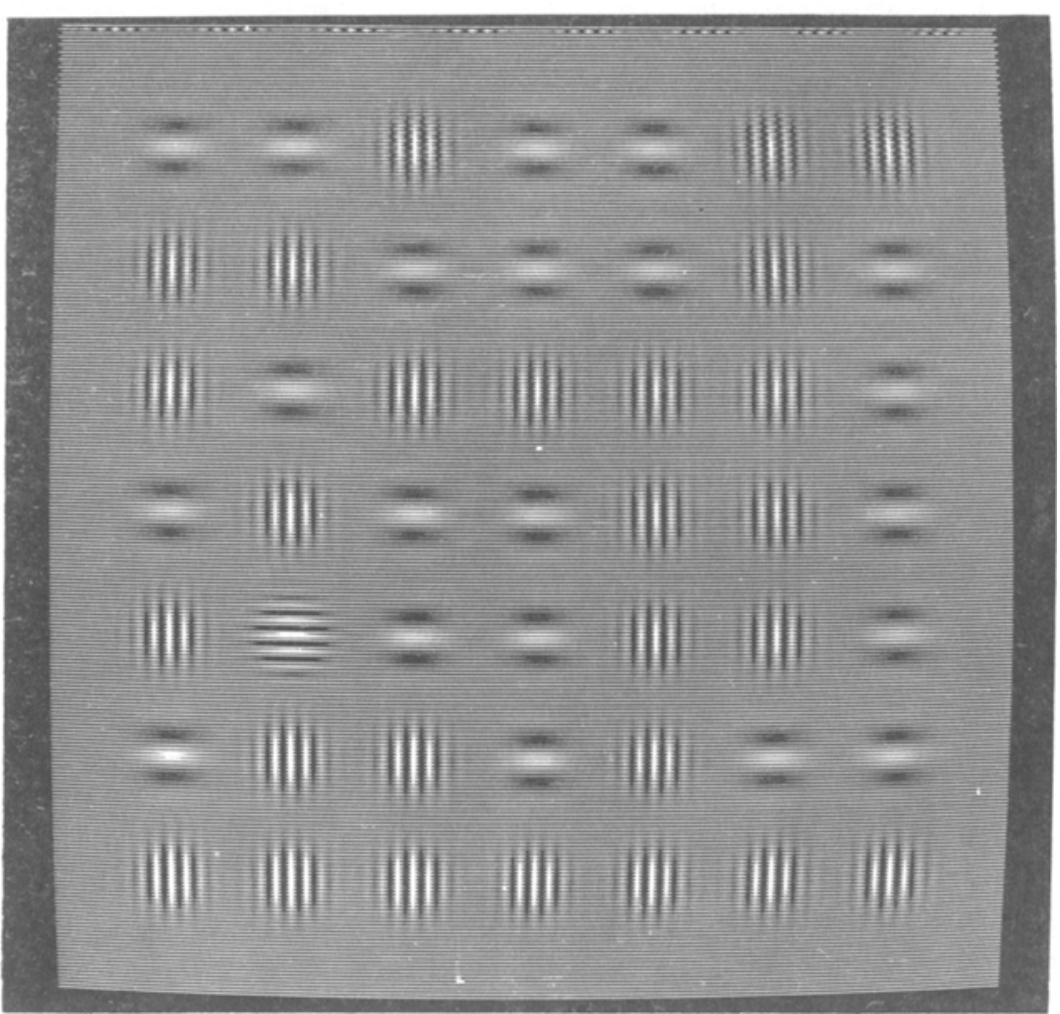

Figure 1. A Treisman-type display in which the targets to be detected are vertical low-frequency patches (A) or horizontal high-frequency patches. The distractors are horizontal low-frequency patches and vertical high-frequency patches. Targets do not "pop out," but note that the borders between the two distractor types do pop out. 


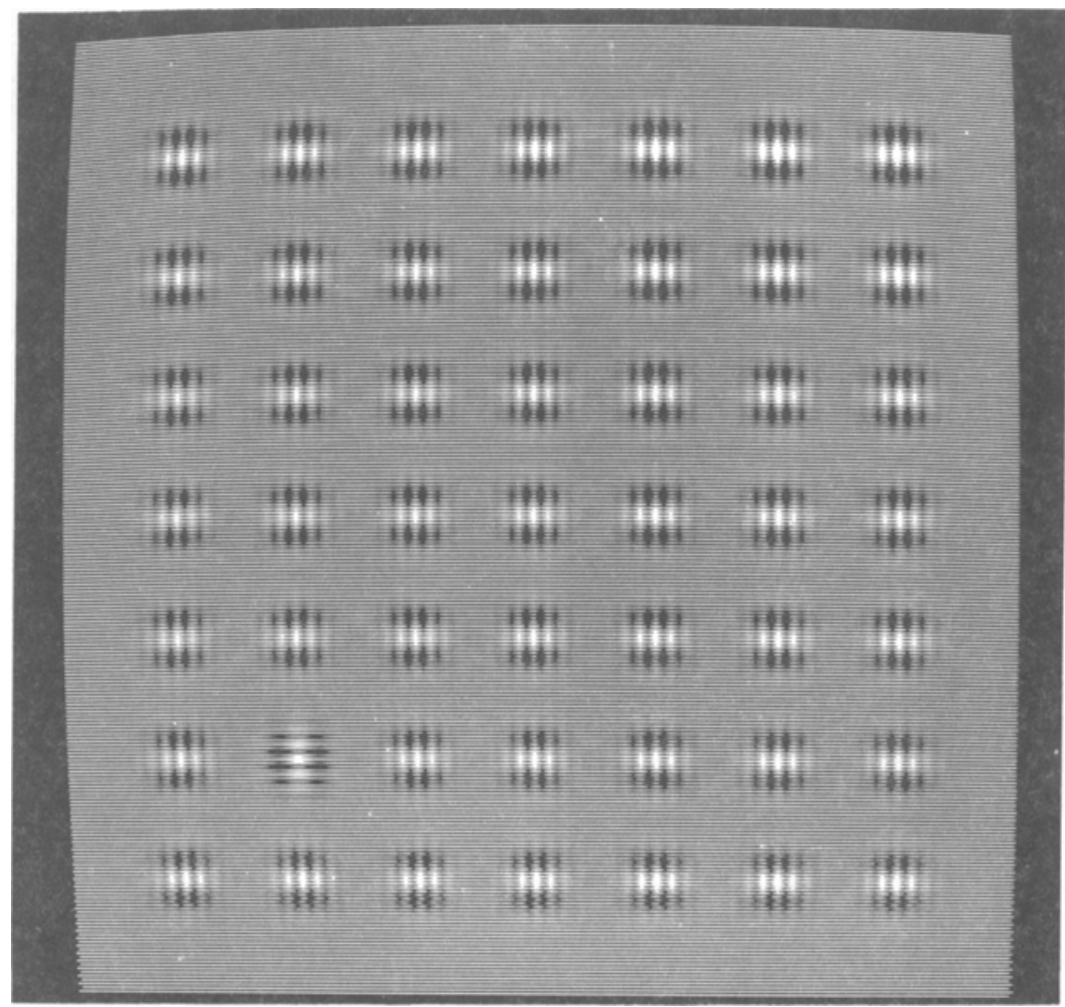

Figure 2. The display of Figure 2 modified to minimize irrelevant feature gradients. The target does pop out now, although their detection requires conjoining spatial frequency and orientation of the individual Gabor patches that generate each texture element.

would have to occur over both space and dimensions (over all neighbors and all feature differences), and would not necessarily be linear. Its properties are under investigation.

\section{REFERENCES}

Bergen, J. R., \& Julesz, B. (1983). Parallel versus serial processing in rapid pattern discrimination. Nature, 303, 696-698.

Houk, M. R., Hofrman, J. E. (1987). Conjunction of color and form without attention: Evidence from orientation-contingent color aftereffect. Joumal of Experimental Psychology: Human Perception \& Performance, 12, 186-199.

Nakayama, K., Silverman, G. H. (1986). Serial and parallel processing of visual feature conjunctions. Nature, 320, 264-265.

NothDURFT, H. C. (1985). Sensitivity for structure gradients in texture discrimination tasks. Vision Research, 25, 1957-1968.
SAGI, D., \& JULESZ, B. (1985). "Where" and "what" in vision. Science, 228, 1217-1219.

SAGI, D., Julesz, B. (1987). Short range limitations on detection of feature differences. Spatial Vision, 2, 39-49.

Treisman, A., \& Gelade, G. (1980). A feature integration theory of attention. Cognitive Psychology, 12, 97-136.

Walters, D., Biederman, I., \& Weisstein, N. (1983). The combination of spatial frequency and orientation is not effortlessly perceived. Supplement to Investigative Ophthalmology \& Visual Science, 24, 238.

WATSON, A. B. (1983). Detection and recognition of simple spatial forms. In O. J. Braddick \& A. C. Sleigh (Eds.), Physical and biological processing of images (pp. 100-114). Berlin: Springer-Verlag. WILSON, H. R. (1983). Psychophysical evidence for spatial channels. In O. J. Braddick \& A. C. Sleigh (Eds.), Physical and biological processing of images (pp. 88-99). Berlin: Springer-Verlag.

(Manuscript received October 28, 1987; revision accepted for publication November 2, 1987.) 